\title{
Sistema compatible de ahusamiento-volumen para Pinus pseudostrobus Lindl. en el ejido Corona del Rosal, Nuevo León, México
}

\section{Compatible taper-volume system for Pinus pseudostrobus Lindl. in the Corona del Rosal ejido, Nuevo León, Mexico}

\author{
Edgar Alan Flores Morales', Analí Celene Rodríguez Alemán', Oscar Alberto Aguirre Calderón", \\ Eduardo Alanís Rodríguez' y Gerónimo Quiñonez Barraza²
}

1 Universidad Autónoma de Nuevo León. Facultad de Ciencias Forestales. Linares, Nuevo León, México.

\author{
2 Instituto Nacional de Investigaciones Forestales \\ Agrícolas y Pecuarias. Campo Experimental Valle de \\ Guadiana. Durango, Durango, México.
}

\section{RESUMEN}

Una de las actividades más importantes en el manejo de los recursos forestales es la extracción de volumen maderable, siendo la variable de mayor importancia debido al valor económico que tiene. El objetivo del trabajo fue ajustar modelos de ahusamiento-volumen para Pinus pseudostrobus Lindl. en el ejido Corona del Rosal, municipio de Galeana, Nuevo León, México. Se emplearon 968 pares de datos de 82 árboles, provenientes de una medición no destructiva utilizando el dendrómetro Criterion RD1000 ${ }^{\circledR}$. El modelo segmentado de Fang resultó ser el más preciso para la especie, se obtuvo un coeficiente de determinación ajustado de 0.98 para el diámetro a diferentes secciones sobre el fuste y 0.97 para el volumen comercial; en los estadísticos raíz de error medio cuadrático y coeficiente de variación, el sistema superó a los modelos de Max y Burkhart y Parresol. La forma dendrométrica de neiloide a paraboloide presentó puntos de inflexión entre $6 \%$ y 9\%, mientras que el cambio de paraboloide a cono ocurre entre $88 \%$ y $91 \%$ de la altura total. Los modelos fueron corregidos por autocorrelación y heterocedasticidad para disminuir el error estándar de los parámetros. Los resultados continuaron siendo favorables para el sistema Fang para predecir diámetros a cualquier altura y volumen a cualquier diámetro, lo que constituye un apoyo para la toma de decisiones en el desarrollo de las actividades forestales.

PALABRAS CLAVE: autocorrelación, coeficiente de determinación ajustado, coeficiente de variación, Criterion RD1000 ${ }^{\circledR}$, heterocedasticidad, sesgo promedio.

\section{ABSTRACT}

One of the most important activities in the management of forest resources is the extraction of wood, this being the most important variable due to its economic value. The objective of the work was to adjust taper-volume models for Pinus psendostrobus Lindl. in the Corona del Rosal ejido in southern Nuevo León, Mexico. 968 pairs of data from 82 trees, coming from non-destructive measurement methods, were employed using the Criterion RD1000 ${ }^{\circledR}$ dendrometer. Fang's segmented model turned out to be the most accurate for the species studied, obtaining an adjusted coefficient of determination of 0.98 for the diameter at different sections on the stem and 0.97 for the commercial volume; in the root mean square error and coefficient of variation statistics, the system surpassed the Max and Burkhart and Parresol models. The dendrometric shape from neiloid to paraboloid presented similar inflection points between $6 \%$ and $9 \%$, while the change from paraboloid to cone occurs between $88 \%$ and $91 \%$ of the total height. The models were corrected for autocorrelation and heteroscedasticity to decrease the standard error of the parameters. The results continued to be favorable for the Fang system to predict diameters at any height and volume at any diameter, which constitutes a support for decision-making in the development of forestry activities.

KEYWORDS: autocorrelation, adjusted coefficient of determination, coefficient of variation, Criterion RD1000 ${ }^{\circledR}$, heterocedasticity, average bias. 


\section{INTRODUCCIÓN}

A escala mundial, uno de los elementos más importantes en la evaluación de recursos forestales es la determinación del volumen existente dentro de un rodal (Cailliez y Alder, 1980). Debido a la dificultad que existe en su medición directa, el volumen se estima en forma indirecta tomando solo una muestra representativa de árboles medidos por métodos destructivos y no destructivos (Alder, 1980). En la actualidad se está optando más por utilizar métodos no destructivos debido a la facilidad y ventajas que se tienen sin tener un impacto que afecte a los bosques, esto sirve tanto para la evaluación de las propiedades de los productos como para su cuantificación (García et al., 2019).

A través de expresiones matemáticas como las funciones de ahusamiento y volumen comercial, esta variable se puede estimar de forma confiable (Prodan, Peters, Cox y Real, 1997). El ahusamiento de un árbol es el coeficiente de estrechamiento del diámetro a lo largo del fuste, por lo que es posible estimar el diámetro a cualquier altura y la altura a cualquier diámetro a través de una ecuación (Clutter, Fortson, Pienaar, Brister y Bailey, 1983; Corral, Chaidez y Sánchez, 1999). Una vez definida la ecuación de ahusamiento, es posible, mediante integración matemática, obtener estimaciones del volumen total o parcial del fuste a cualquier límite de longitud o diámetro mínimo deseado (Lynch, Chang y Chandler, 1992).

Las ecuaciones de ahusamiento van desde funciones simples (Demaerschalk, 1972; McTague y Bailey, 1987; Zakrzewski y MacFarlane, 2006) hasta complejas, como las funciones segmentadas (Max y Burkhart, 1976; Fang et al., 2000; Jiang, Brooks y Wang, 2005; Hernández-Ramos, Hernández-Ramos, García-Cuevas, Martínez-Ángel, Tamarit Urias y García Espinoza, 2018). Las funciones de ahusamiento simples describen con menor precisión el perfil del fuste, mientras que las funciones segmentadas proporcionan estimaciones de diámetros con sesgos menores en la mayor parte del fuste y son más precisas para estimar el volumen total y comercial, por lo que se hace indispensable ajustar el modelo para distribuir de forma porcentual el volumen comercial. Por otra parte, permiten describir los tipos dendrométricos del árbol: neiloide en la sección inferior, cilindro o paraboloide en la sección media y cónica en la sección superior (Martínez-Ángel, De los Santos Posadas, González, Cruz-Cobos y QuiñonesBarraza, 2019). Si bien, ya se han desarrollado modelos de ahusamiento para algunas especies y regiones de México, aún falta generar modelos para especies de interés comercial como Pinus pseudostrobus Lindl. en el sur de Nuevo León.

Pinus psendostrobus Lindl. destaca por el valor comercial de su madera en el mercado, la cual es considerada de buena calidad y se utiliza, principalmente, para el aserrío (Estrada et al., 2014). Al igual que otras especies, su distribución es muy amplia dentro del territorio nacional (Flores-Morales, Aguirre-Calderón, Quiñónez-Barraza, González-Tagle y Jiménez-Pérez, 2019).

\section{MATERIALES Y MÉTODOS}

\section{Descripción del área de estudio}

El ejido Corona del Rosal se ubica en el municipio de Galeana, al sur del estado de Nuevo León. Administrativamente se encuentra dentro del área de influencia del Distrito de Desarrollo Rural Galeana y pertenece a la Unidad de Manejo Forestal (UMAFOR) 1901 (Fig. 1).

La altitud promedio del ejido Corona del Rosal es de 1620 m s.n.m.; los puntos con mayor altitud se encuentran en parajes conocidos como "El Infiernillo", "Las Bancas" y "La Zacatosa", que alcanzan hasta 3160 m s.n.m. (Unidad de Conservación y Desarrollo Forestal No. 1 [UCDF], 1996).

El área presenta un clima templado con verano cálido, la temperatura media anual varía entre $12{ }^{\circ} \mathrm{C}$ y $18{ }^{\circ} \mathrm{C}$, mientras que la temperatura del mes más frío oscila entre $3{ }^{\circ} \mathrm{C}$ y $12{ }^{\circ} \mathrm{C}$. El régimen de lluvias es escaso la mayor parte del año, con un porcentaje de lluvias invernales menor a $18 \%$ con respecto al total anual (UCDF, 1996).

Predominan los suelos someros, mismos que se encuentran en diversos grados de asociación, entre los que se distinguen los grupos Litosol, Rendzina y Regosol con una clase de textura media (UCDF, 1996). 


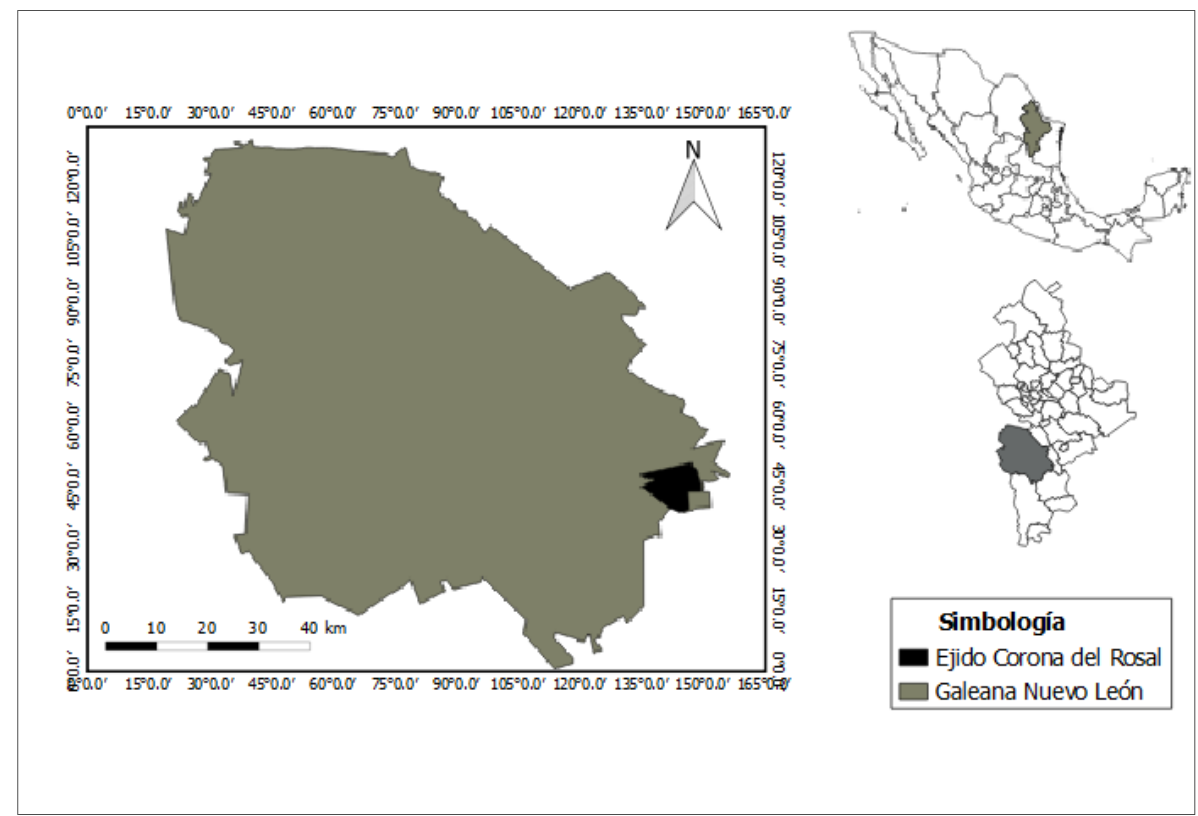

FIGURA 1. Ubicación del área de estudio.

\section{Variables utilizadas para el ajuste en los modelos}

Las variables utilizadas se presentan a continuación:

$\mathrm{d}=$ diámetro a una altura determinada $(\mathrm{cm})$

$\mathrm{D}=$ diámetro normal $(\mathrm{cm})$

$\mathrm{h}=$ altura (m), desde la base del árbol hasta el punto donde se alcanza un diámetro dado

$\mathrm{H}=$ altura total del árbol (m)

$\mathrm{Vt}=$ volumen total del fuste $\left(\mathrm{m}^{3}\right)$

$\mathrm{Vc}=$ volumen comercial $\left(\mathrm{m}^{3}\right)$, a un diámetro dado

\section{Caracterización de la base de datos}

La base de datos utilizada fue de 968 mediciones de diámetro a diferentes alturas obtenida de 82 árboles para $P$. pseudostrobus. Estos datos provienen de mediciones hechas con el dendrómetro $\mathrm{RD} 1000^{\circledR}$, los árboles fueron seleccionados de acuerdo con las características diamétricas del área de estudio, se consideró cubrir todas las categorías existentes.

Para la toma de datos se midió cada ejemplar con el dendrómetro para obtener sus diámetros a diferentes alturas. La primera a la altura mínima posible de corte del tocón; se tomaron tres secciones hasta llegar al diámetro normal $(1.30 \mathrm{~m})$, las dos primeras de $30 \mathrm{~cm}$ de altura y la tercera de $70 \mathrm{~cm}$. Posteriormente, se obtuvieron secciones de $2 \mathrm{~m}$ de longitud de manera subsecuente hasta la punta del árbol. Por individuo, se midió el diámetro normal, la longitud total y de cada sección los diámetros y las longitudes. Los volúmenes de las trozas se calcularon con la fórmula de Smalian (1) y la parte final con la del cono (2)

$$
\begin{gathered}
V=\frac{S 1+S 2}{2} * h \\
V=\frac{S b * h}{3}
\end{gathered}
$$

Donde:

$\mathrm{S} 1=$ superficie menor $\left(\mathrm{m}^{2}\right)$

$\mathrm{S} 2=$ superficie mayor $\left(\mathrm{m}^{2}\right)$

$\mathrm{Sb}=$ superficie de la base $\left(\mathrm{m}^{2}\right)$

$\mathrm{h}=$ longitud $(\mathrm{m})$

$\mathrm{V}=\operatorname{volumen}\left(\mathrm{m}^{3}\right)$

En la tabla 1 se presenta el resumen general de las variables de la base de datos usada en el ajuste de los sistemas compatibles de ahusamiento y volumen comercial, el cual presenta los estadísticos descriptivos. 
TABLA 1. Valores de los estadísticos descriptivos de la base de datos utilizada de Pinus pseudostrobus.

\begin{tabular}{lcccccc}
\hline & $H$ & $d$ & $h$ & $D$ & $V_{c}$ & $V_{t}$ \\
\hline N & 82 & 968 & 968 & 82 & 968 & 82 \\
MIN & 8.40 & 0 & 0.30 & 14.90 & 0.0068 & 0.0945 \\
MAX & 30.70 & 78.80 & 28.20 & 66.60 & 6.1693 & 6.1693 \\
MED & 20.02 & 30.28 & 8.99 & 41.56 & 1.1065 & 1.6691 \\
DESV & 4.63 & 17.62 & 7.07 & 15.27 & 1.1639 & 1.3828 \\
EST & & & & & & \\
\hline
\end{tabular}

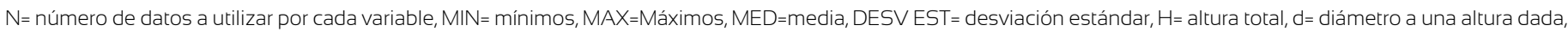
$\mathrm{h}=$ altura $\mathrm{a}$ un diámetro dado, $\mathrm{D}=$ diámetro normal, $\mathrm{V}_{\mathrm{c}}=$ volumen comercial y $\mathrm{V}_{\mathrm{t}=\text { volumen total }}$

Se examinaron los gráficos de dispersión entre diámetro relativo y altura relativa que son, respectivamente, el cociente entre el diámetro $\mathrm{d}$ a la altura $\mathrm{h}$ y el diámetro normal D, y el cociente entre h y la altura total $\mathrm{H}$ del árbol, así como el de volumen acumulado para la detección de datos atípicos, ya que puede haber errores en la toma de información (Martínez-Ángel et al., 2019; Gómez-García et al., 2016). A continuación, se muestra el comportamiento entre diámetro relativo $(\mathrm{d} / \mathrm{Dn})$ y altura relativa $(\mathrm{h} / \mathrm{H})$ y del volumen acumulado respecto a la altura del fuste sin información errónea y tendencia lógica (Fig. 2).

\section{Modelos utilizados}

Se usaron los modelos segmentados de ahusamiento (d) y volumen comercial $\left(\mathrm{v}_{\mathrm{c}}\right)$ ajustados como sistema compatible, desarrollados por Fang et al. (2000), Max y Burkhart (1976) y Parresol, Hotvedt y Cao (1987), estos se han utilizado en gran medida en diferentes investigaciones como la de Tamarit-Urias, Rojas-Díaz, Quiñonez-Barraza, OrdoñezPrado y Monárrez- González (2017), ya que son los que presentan los mejores resultados (Tabla 2).

\section{Método de ajuste}

El ajuste estadístico se realizó con la técnica de regresión aparentemente no relacionada (SUR) con el procedimiento MODEL del paquete estadístico SAS/ETS®) (Statistical Analysis Sistem Institute Inc., 2013), ya que proporciona una mejor estimación y reduce el error estándar de los parámetros para un sistema de ecuaciones; además permite la compatibilidad total dentro del sistema (Tamarit-Urias et al., 2017; García et al., 2019; Martínez-Ángel et al., 2019).

\section{Estadísticos para medir el ajuste de los modelos}

Los estadísticos y criterios que se emplearon para la selección y comparación de los modelos son los siguientes: raíz del error medio cuadrático (REMC), coeficiente de variación $(\mathrm{CV}, \%)$, coeficiente de determinación ajustado $\left(\mathrm{R}^{2}{ }_{\text {adj }}\right)$, sesgo promedio absoluto $(\bar{E})$, criterio de información Akaike (AIC) y análisis gráfico de residuales (Hernández-Ramos et al., 2017a; López, Molina, Barrios y Aguirre (2018) (Tabla 3).

\section{Autocorrelación y heterocedasticidad}

Para corregir la autocorrelación se aplicó un modelo autorregresivo de tiempo continuo (CAR (X)) en el componente de ahusamiento, que considera la distancia entre mediciones de la altura comercial en cada árbol (Quiñonez-Barraza, García-Espinoza y Aguirre-Calderón, 2018; Bautista, 2016), la expresión es:

$e_{i j}=d_{1} y_{1} h_{i j}-h_{i j-1} \quad e_{i j-_{1}}+d_{2} y_{2}{ }^{h_{i j}-h_{i j-2}} \quad e_{i j-2}+E_{i j}$

Donde:

$e_{i j}=j$-ésimo residual ordinario en el árbol $i$

$d_{i}=1 \operatorname{para} j>1 ; d_{\mathrm{i}}=0 \operatorname{para} j=1$

$y_{1}=$ parámetro autorregresivo de orden $i$

$h_{i j}-h_{i j-1}=$ distancia de separación entre la j-ésima observación y la observación previa dentro de cada árbol 


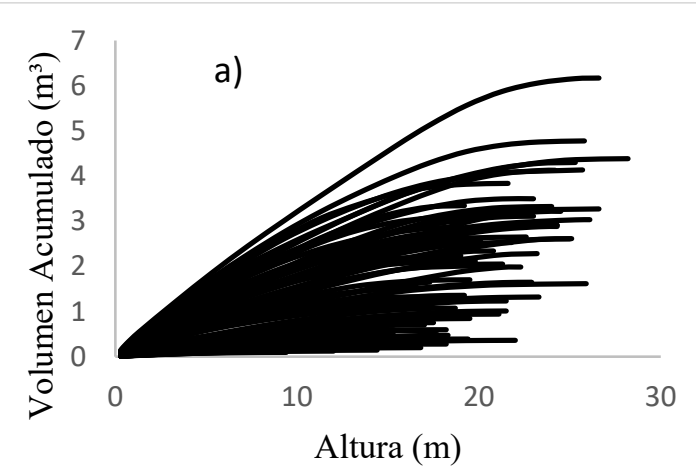

FIGURA 2. Volumen acumulado respecto a la altura del fuste de la especie en estudio (a) y diámetro relativo (d/Dn) contra altura relativa $(\mathrm{h} / \mathrm{H})(\mathrm{b})$.

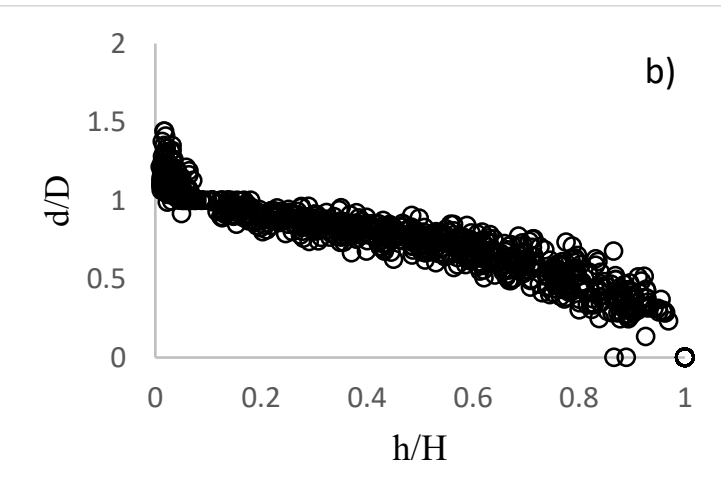

TABLA 2. Sistemas de ahusamiento y volumen ajustados para Pinus pseudostrobus Lindl.

Ecuación de ahusamiento

Ecuación de volumen comercial

$$
\begin{array}{cc}
\text { Fang et al. (2000) } & C_{1}=\left(\frac{\alpha^{0} D^{\alpha_{1}} H^{\alpha_{2}-\frac{k}{\beta_{1}}}}{\beta_{1}\left(R_{o}-R_{1}\right)+\beta_{2}\left(R_{1}-\alpha_{1} R_{2}\right)+\beta_{3} \alpha_{1} R_{2}}\right)^{1 / 2} \\
d=c_{1}\left[H^{\frac{K-\beta_{1}}{\beta_{1}}}(1-q)^{\frac{k-\beta}{\beta}} \alpha_{1}^{I_{1}+I_{2}} \alpha_{2}^{I_{2}}\right]^{1 / 2} & \alpha_{1}=\left(1-p_{1}\right)^{\frac{K\left(\beta_{2}-\beta_{1}\right)}{\beta_{1} \beta_{2}}} \alpha_{2}=\left(1-p_{2}\right)^{\frac{K\left(\beta_{3}-\beta_{2}\right)}{\beta_{2} \beta_{3}}} \\
\text { donde }\left\{\begin{array}{l}
I_{1}=1 \text { sip } p_{1} \leq q \leq p_{2} ; \text { de lo contrario 0 } \\
I_{2}=1 \text { sip } p_{2} \leq q \leq 1 ; \text { de lo contrario } 0
\end{array}\right. & V_{c}=C_{1}^{2} H^{\frac{k}{\beta_{1}}}\left[\beta_{1} R_{o}+\left(I_{1}+I_{2}\right)+\left(\beta_{2}-\beta_{1}\right) R_{1}+I_{2}\left(\beta_{3}-\beta_{2}\right) \alpha_{1} R_{2}\right. \\
R_{0}=\left(1-H_{B} / H\right)^{K / \beta_{1}} R_{1}=\left(1-P_{1}\right)^{K / \beta_{1}} & \left.-\beta(1-q)^{\frac{k}{\beta}} \alpha_{1}^{I_{1}+I_{2}} \alpha_{2}^{I_{2}}\right]
\end{array}
$$

Max y Burkhart (1976)

$d=D\left[\begin{array}{c}\beta_{1}(q-1)+\beta_{2}\left(q^{2}-1\right)+\beta_{3}\left(P_{1}-q\right)^{2} I_{1}+ \\ \beta_{4}\left(P_{2}-q\right)^{2} I_{2}\end{array}\right]^{1 / 2}$

$$
\begin{gathered}
V_{c}=k D^{2} H\left\{\frac{\beta_{2}}{3}\left(q^{3}-z_{1}^{3}\right)+\frac{\beta_{1}}{2}\left(q^{2}-z_{1}^{2}\right)-\left(\beta_{1}+\beta_{2}\right)\left(q-z_{1}\right)\right. \\
-\frac{\beta_{3}}{3}\left[\left(p_{1}-q\right)^{3} J_{1}-\left(p_{1}-z_{1}\right)^{3} K_{1}\right]
\end{gathered}
$$

donde $\left\{\begin{array}{l}I_{1}=1 \text { si } q \leq p_{1} ; \text { de lo contrario } 0 \\ I_{2}=1 \text { si } q \leq p_{2} ; \text { de lo contrario } 0\end{array}\right.$

$$
\left.-\frac{\beta_{4}}{3}\left[\left(p_{2}-q\right)^{3} J_{2}-\left(p_{2}-z_{1}\right)^{3} K_{2}\right]\right\}
$$

$$
q=H M / H
$$

$$
\text { donde }\left\{\begin{array}{c}
J_{1}=1 \mathrm{siq} \leq p_{1} ; J_{2}=0 \mathrm{siq}>p_{2} \\
K_{1}=1 \mathrm{siz} z_{1} \leq p_{1} ; K_{2}=0 \mathrm{siz}_{1}>p_{1}
\end{array}\right.
$$

Parresol et al. (1987)

$$
\begin{gathered}
\mathrm{d}=D \sqrt{X^{2}\left(\beta_{1}+\beta_{2} X\right)+\left(X-p_{1}\right)^{2}\left[\beta_{3}+\beta_{4}\left(X+2 p_{1}\right) I_{1}\right]} \\
\text { donde } I_{1}=1 \text { si } z \geq p_{1} ; \text { de lo contrario } 0
\end{gathered}
$$

$$
\begin{gathered}
V_{c}=k D^{2} H\left\{\left[\left(\frac{\beta_{2}+\beta_{4} I_{1}}{4\left(X^{4}-z_{b}^{4}\right)}\right)+\left(\frac{\beta_{1}+\beta_{3} I_{1}}{3\left(X^{3}-z_{b}{ }^{3}\right)}\right)+\left(\frac{2 \beta_{1}\left(p_{1} I_{1}\right)+\left(2 p_{1} \beta_{4}\right)}{2\left(X^{2}-z_{b}^{2}\right)}\right)\right.\right. \\
\left.\left.+\left(\left(p_{1}^{2} I_{1} \beta_{3}\right)+\left(2 p_{1}-\beta_{4}\right)\left(X-z_{b}\right)\right)\right]\right\}
\end{gathered}
$$

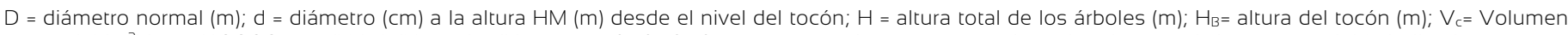
comercial $\left(m^{3}\right) \mathrm{k}=\pi / 40000 ; z_{b}=\left(H-h_{b}\right) / H ; z_{1}=h_{b} / H ; \alpha_{0}, \alpha_{1}, \alpha_{2}, \beta_{1}, \beta_{2}, \beta_{3}, \beta_{4}, P_{1}, P_{2}$ son parámetros que serán estimados por el sistema; I variable indicadora. 
TABLA 3. Estadísticos evaluados para la selección de la ecuación de mejor ajuste.

\begin{tabular}{|c|c|}
\hline Estadístico & Expresión matemática \\
\hline Raíz del error cuadrado medio (REMC) & $R E M C=\sqrt{\frac{\left[\sum_{i=1}^{n}\left(Y_{i}-\widehat{Y}_{l}\right)^{2}\right]}{n-p}}$ \\
\hline Coeficiente de variación (CV) & 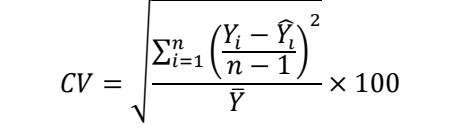 \\
\hline Coeficiente de determinación Ajustado $\left(\mathbf{R}_{\mathbf{A d j}}^{2}\right)$ & $R^{2} a d j=1-\left[\frac{\sum_{i=1}^{n}\left(Y_{i}-\widehat{Y}_{l}\right)^{2}}{\sum_{i=1}^{n}\left(Y_{i}-\bar{Y}_{l}\right)^{2}}\right]\left(\frac{n-1}{n-p}\right)$ \\
\hline Sesgo promedio absoluto $(\overline{\boldsymbol{E}})$ & $\bar{E}=\left[\frac{\sum_{i=1}^{n}\left(Y_{i}-\widehat{Y}_{\imath}\right)}{n}\right]$ \\
\hline Criterio de información de Akaike (AIC) & $A I C=n \ln \left[\sum_{i=1}\left(Y_{i}-\widehat{Y}_{l}\right)^{2} / n\right]+2 p$ \\
\hline
\end{tabular}

$\mathrm{Y}_{\mathrm{i}}, \widehat{Y}_{1}, \bar{Y}$ son los valores observados, estimados y promedio del ahusamiento (d) y volumen comercial $(\mathrm{Vc}$ ) respectivamente, $\mathrm{n}$ es el número total de observaciones para el ajuste de los sistemas $Y_{\mathrm{i}}, \mathrm{p}$ es el número de parámetros del sistema.

Para corroborar la corrección de autocorrelación, se examinaron los resultados de la prueba de Durbin-Watson (DW); valores pequeños indican alta correlación positiva (cercanos a cero); con próximos a 4, los residuos son correlacionados negativamente; lo deseable es que los valores sean cercanos a 2 , es decir que los residuos son independientes (Quiñones- Barraza et al., 2014; Pérez, 2003; Linnell, 1996; Draper y Smith, 1998).

El problema de heterocedasticidad del ahusamiento y volumen comercial fue corregido con una función de potencia de la varianza del residual $\sigma_{i}^{2}\left(D^{2} H\right)^{\varnothing}$ con el $D$ y la $H$ como variables independientes (Crecente-Campo, Rojo y Diéguez-Aranda, 2009). El valor $\emptyset$ se obtuvo por el método sugerido por Harvey (1975), que consiste en el uso del error estimado de modelo sin pesos $\hat{e}_{i}$ como la variable dependiente en la varianza del error del modelo $\hat{e}_{\boldsymbol{i}}^{2}=$ $\emptyset_{0}\left(D^{2} H\right)^{\emptyset_{1}}$.

\section{RESULTADOS}

Los estadísticos y los resultados de la prueba de DurbinWatson (DW) obtenidos del ajuste simultáneo para las ecuaciones ahusamiento y volumen comercial se presentan en la tabla 4.

Los modelos de Fang, Borders y Bailey (2000) y Max y Burkhart (1976) presentaron resultados similares con una $\mathrm{R}^{2}$ adj de 0.97 para el volumen, mientras que para el modelo de ahusamiento tuvo mejor ajuste el modelo de Fang et al. (2000) con una $\mathrm{R}^{2}$ adj de 0.98. Después de la corrección por auto correlación, los resultados de la prueba DurbinWatson fueron similares, por lo que se asume que los modelos no presentan autocorrelación, la RCME y el sesgo se mantuvieron pequeños de manera similar para los tres modelos.

La tabla 5 muestra los valores de los parámetros y de los puntos de inflexión ( $p 1$ y $p 2$ ). Para el primer sistema (Fang et al., 2000) p1 ocurre a 6.1\% de la altura mínima del tocón y $p 2$ ocurre a $91 \%$ de la altura relativa sobre el fuste. Para el modelo de Max y Burkhart (1976), los segmentos se presentan a $9 \%$ para $\not 1$ y $\not 2$ se fijó a $88 \%$ para lograr una convergencia en el sistema. El modelo de Parresol et al. (1987) solo cuenta con un punto de inflexión lo cual lo explica $65 \%$ de la altura relativa. 
TABLA 4. Valores de los estadísticos de las ecuaciones compatibles de ahusamiento-volumen ajustadas para Pinus pseudostrobus.

\begin{tabular}{|c|c|c|c|c|c|c|c|}
\hline Sistema & Variable & REMC & $C V$ & $R^{2} a d j$ & Sesgo & $A I C$ & $D W$ \\
\hline \multirow[t]{2}{*}{1} & Vc & 0.17 & 15.33 & 0.97 & 0.02 & -3392.47 & 1.80 \\
\hline & d & 2.12 & 6.93 & 0.98 & 0.24 & 1465.26 & \\
\hline \multirow[t]{2}{*}{2} & Vc & 0.17 & 15.49 & 0.97 & 0.03 & -3355.72 & 1.85 \\
\hline & d & 2.22 & 7.27 & 0.96 & 0.17 & 1547.66 & \\
\hline \multirow[t]{2}{*}{3} & Vc & 0.49 & 43.50 & 0.81 & 0.11 & -1355.31 & 1.83 \\
\hline & d & 3.18 & 10.51 & 0.96 & 0.07 & 2251.25 & \\
\hline
\end{tabular}

Sistema = 1) Fang et al. (2000), 2) Max y Burkhart (1976), 3) Parresol (1987); Vc= Volumen comercial; d= Diámetro a una altura dada; RCME= Raíz cuadrado medio del error; $\mathrm{CV}=$ Coeficiente de variación; R2adj= Coeficiente de determinación ajustado; $\mathrm{AIC}=$ Criterio de Akaike; $\mathrm{DW}=$ Prueba durbin-Watson.

TABLA 5. Valores de los parámetros de las ecuaciones compatibles de ahusamiento-volumen ajustadas para Pinus pseudostrobus.

\begin{tabular}{|c|c|c|c|c|c|c|c|c|c|}
\hline & & 1 & & & 2 & & & 3 & \\
\hline Parámetro & Estimador & $\mathrm{EE}$ & $\operatorname{Pr}>\mid \mathrm{tl}$ & Estimador & EE & $\operatorname{Pr}>\mid \mathrm{tl}$ & Estimador & $\mathrm{EE}$ & $\operatorname{Pr}>|t|$ \\
\hline$\alpha O$ & 0.000067 & 0.0000048 & $<0.0001$ & & & & & & \\
\hline$\alpha 1$ & 1.904765 & 0.0217 & $<0.0001$ & & & & & & \\
\hline$\alpha 2$ & 0.97890 & 0.0287 & $<0.0001$ & & & & & & \\
\hline$\beta 1$ & 0.00001 & 0.0000005 & $<0.0001$ & -3.9175 & 0.0201 & $<0.0001$ & 3.4436 & 0.1052 & $<0.0001$ \\
\hline$\beta 2$ & 0.00004 & 0.0000003 & $<0.0001$ & 50.8718 & 6.6743 & $<0.0001$ & -2.4112 & 0.1418 & $<0.0001$ \\
\hline$\beta 3$ & 0.00006 & 0.0000068 & $<0.0001$ & -1.8041 & 0.0424 & $<0.0001$ & -0.6372 & 0.1741 & 0.0003 \\
\hline$\beta 4$ & & & & & & & 1.3299 & 0.1755 & $<0.0001$ \\
\hline $\mathrm{pl}$ & 0.061396 & 0.00299 & $<0.0001$ & 0.0960 & 0.0062 & $<0.0001$ & & & \\
\hline $\mathrm{p} 2$ & 0.913774 & 0.0102 & $<0.0001$ & 0.8840 & & & 0.6557 & 0.0887 & $<0.0001$ \\
\hline $\mathrm{yl}$ & 0.912786 & 0.0208 & $<0.0001$ & 0.8968 & 0.0227 & $<0.0001$ & 0.9497 & 0.0218 & $<0.0001$ \\
\hline y2 & 0.760348 & 0.0211 & $<0.0001$ & 0.7344 & 0.0243 & $<0.0001$ & 0.8164 & 0.0197 & $<0.000$ \\
\hline
\end{tabular}



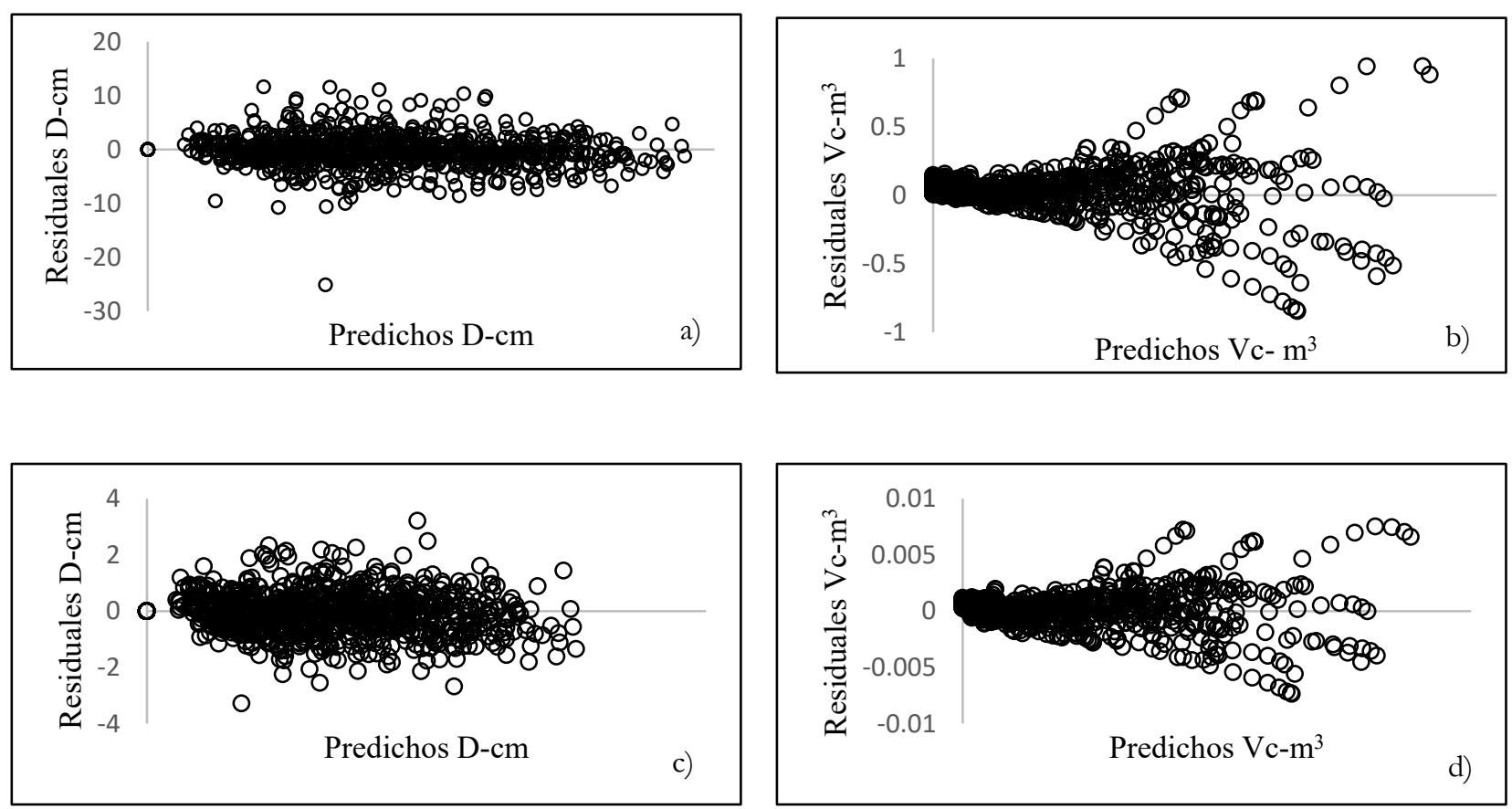

FIGURA 3. Gráfica de residuales de ahusamiento contra los valores predichos de diámetro (a y b) y volumen comercial (c y d) del sistema Fang et al. (2000) sin corrección y con corrección por heterocedasticidad en Pinus pseudostrobus.

Es común que la mayor parte de los modelos de volumen sufran problemas de heterocedasticidad dado que, a valores más altos de diámetro y altura, la variación en los volúmenes de los árboles se hace más grande (Torres y Magaña, 2001). Los residuos antes y después de la corrección por autocorrelación y heterocedasticidad se observan en la figura 3. La corrección por heterocedasticidad fue adecuada en el modelo de Fang et al. (2000) y muestra una distribución adecuada de los residuales haciendo la estimación más homogénea y con menor amplitud.

Lo que destaca es la disminución del error estándar de los parámetros al considerar la variabilidad específica del árbol; sin embargo, los valores de los parámetros no difieren de manera considerable (García et al., 2019), aunque corrigieron la violación a los supuestos de regresión (autocorrelación y heterocedasticidad).

El comportamiento de los diámetros observados contra las estimaciones realizadas se muestra en la figura 4 y se puede observar que tienen una tendencia semejante; lo mismo ocurre en el volumen comercial, indicador de un buen ajuste del modelo para la especie en estudio.

Las estimaciones del volumen comercial con la ecuación propuesta presentan buenos ajustes, aunque se tienen problemas al predecir valores en árboles con volúmenes comerciales mayores a $5 \mathrm{~m}^{3}$. En estos casos, hay sesgo en las predicciones; se asume que la causa es a la poca información que se tiene en esta parte de los datos.

\section{DISCUSIÓN}

Los resultados obtenidos del sistema Fang et al. (2000), ajustados con regresión aparentemente no relacionada (SUR), muestran estadísticos adecuados ya que los errores estándar se minimizaron y todos los parámetros fueron significativos, lo que concuerda con Quiñonez-Barraza, De los Santos-Posadas, Héctor, Álvarez-González y Velázquez-Martínez (2014). 


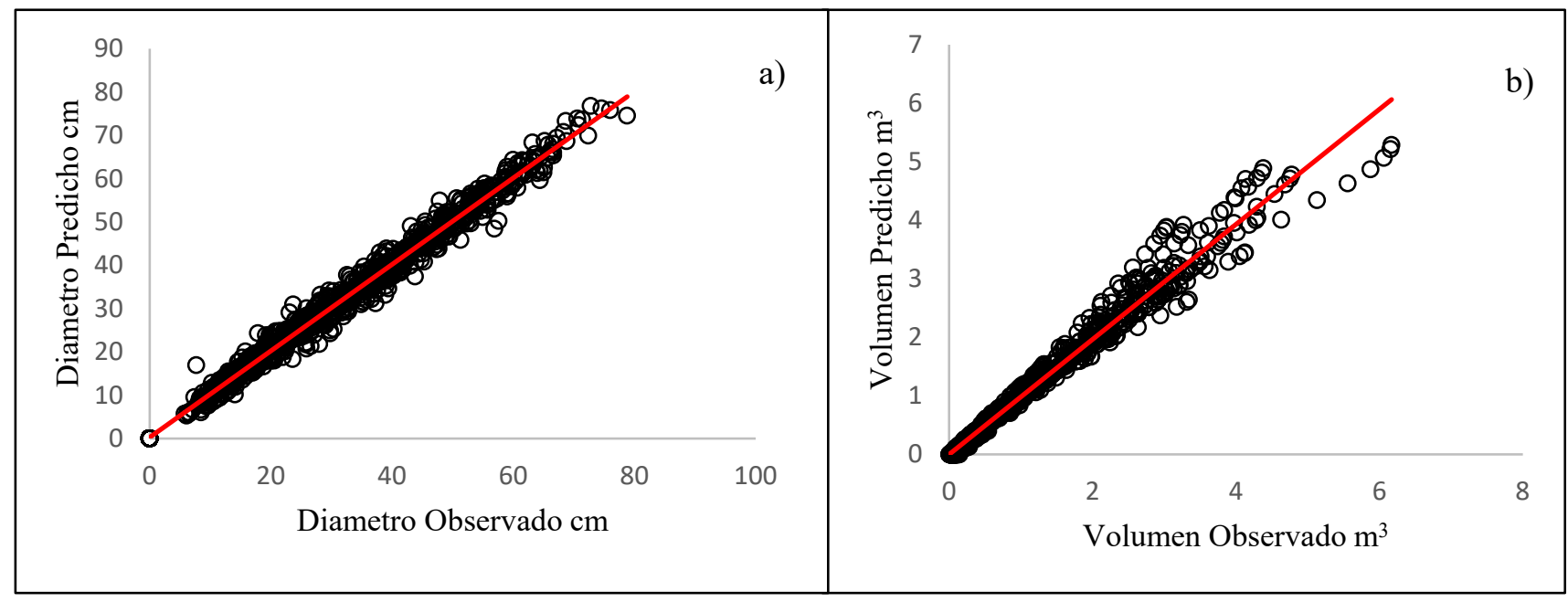

FIGURA 4. Gráficos de diámetro observado contra diámetro predicho (a) y volumen observado contra volumen predicho (b).

Los estadísticos obtenidos del sistema compatible de ahusamiento-volumen fueron similares a los presentados por García et al. (2019), quienes obtuvieron valores de $\mathrm{R}^{2}$ de 0.98 para la ecuación de ahusamiento y de 0.97 para la ecuación de volumen en Pinus pseudostrobus Lindl. en San Juan Parangaricutiro, Michoacán. Los puntos de inflexión se asemejan ya que oscilan para $p 1$ entre $6 \%$ y $8 \%$ y para $p 2$ entre $88 \%$ y $91 \%$, los valores de la prueba Durbin-Watson se asemejan y son deseables.

Los problemas de heterocedasticidad y autocorrelación se corrigieron, con lo cual se mejoró la distribución de los residuales en todos los sistemas analizados, lo que concuerda con varios autores como Hernández-Ramos et al. (2017b) y Tamarit-Urias et al. (2014).

En términos de calidad de ajuste, se obtuvieron resultados similares en un estudio de Pompa, Corral, Díaz y Martínez (2009), para árboles de Quercus sp. donde el modelo de Fang et al. (2000) describió de manera precisa el perfil fustal y predijo adecuadamente el volumen comercial en el noreste del estado de Chihuahua, México. QuiñonezBarraza et al. (2014) encontraron que el modelo de Fang et al. (2000) fue el que tuvo los mejores resultados para las principales especies de Pinus en Durango, estableciendo parámetros globales con variables indicadoras para todas las especies.
López (2013) y Fierros y Martínez (2013) también concluyen que el modelo de Fang et al. (2000) es el mejor para predecir el perfil fustal, así como el volumen comercial para especies de Pinus en Durango en los municipios de San Dimas y Pueblo Nuevo, Durango. Brooks, Jiang y Ozçelik (2008) seleccionaron el sistema de Max y Burkhart (1976) para calcular los diámetros relativos y los volúmenes de las especies de Pinus brutia, Cedrus libani y Abies cilica en Turquía; el modelo demostró ser el más preciso con base en sus estadísticos de ajuste y mostró un rendimiento constante en la estimación de diámetro y volumen. El modelo de Fang et al. (2000) permite estimar el diámetro a cualquier altura y el volumen a cualquier diámetro, lo cual tiene aplicación en la distribución de productos para determinar el uso más adecuado de la materia prima forestal.

\section{CONCLUSIONES}

De los sistemas compatibles de ahusamiento-volumen, el basado en el modelo de Fang et al. (2000) fue el que tuvo mejores estadísticos para la base de datos utilizada; estos se generaron bajo la técnica de regresión aparentemente no relacionada (SUR), después permitiendo estimar de forma directa el diámetro a cualquier altura y, de la misma manera, referir el volumen a una altura dada o a un diámetro determinado. 
Los estadísticos del modelo muestran que se corrigieron adecuadamente los problemas de heterocedasticidad y autocorrelación, optimizando los valores de los parámetros estadísticos y disminuyendo su error estándar y sesgo.

La confiabilidad del sistema compatible de ahusamiento-volumen de Fang et al. (2000), ajustado con información procedente de métodos no destructivos, se garantiza por la alta precisión en la descripción del perfil fustal para la especie en estudio sobre todo en la sección donde se concentra la mayor cantidad de volumen.

Los sistemas compatibles de ahusamiento y volumen comercial ajustados constituyen una herramienta importante en la planeación del manejo forestal, ya que permiten a los técnicos responsables del manejo de los bosques de la región en estudio una estimación precisa del volumen y de productos maderables.

\section{REFERENCIAS}

Alder, D. (1980). Estimación del volumen forestal y predicción del rendimiento, con referencia especial a los trópicos. Roma, Italia: Organización de las Naciones Unidas para la Agricultura y la Alimentación.

Bautista, A. (2016). Funciones de ahusamiento-volumen para Pinus patula Schl. et cham. en la Región Chinaguapan-Zacatlán Puebla. Tesis de Maestría. Universidad Autónoma de Nuevo León. Linares, Nuevo León, México.

Brooks, J., Jiang, L., \& Ozçelik, R. (2008). Compatible stem volume and taper equations for Brutian pine, cedar of Lebanon, and Cilicica fir in Turkey. Forest Ecology and Management 25(6), 147-151. doi: 10.1016/j.foreco.2008.04.018

Cailliez, F., \& Alder, D. (1980). Estimación del volumen forestal y predicción del rendimiento con referencia especial a los trópicos. Organización de las Naciones Unidas para la Agricultura y la Alimentación.

Clutter, J., Fortson, J., Pienaar, L., Brister, G., \& Bailey, R., L. (1983). Timber management a quantitative approach. New York: Wiley.

Corral, S., Chaidez, J., \& Sánchez, F. (1999). Ajuste de funciones de ahusamiento a los perfiles fustales de cinco pináceas de la región de El Salto, Durango. Madera y Bosques, 5(2), 53-65. doi: $10.21829 /$ myb.1999.521347

Crecente-Campo, F., Rojo A., A., \& Diéguez-Aranda, U. (2009). A merchantable volumen system for Pinus sylvestris $\mathrm{L}$. in the major mountains ranges of Spain. Annals of Forest Science, 66, 808. doi: 10.1051 / forest $/ 2009078$

Demaerschalk, J. (1972). Converting volume equations to compatible taper equations. Forest Science, 18(3), 241-245. doi: 10.1093/forestscience/18.3.241

Draper, N., \& Smith, H. (1998). Applied regression analysis (3 ${ }^{\mathrm{a}}$ ed.). John Wiley \& Sons.

Estrada, A., Villareal, J., Salinas, M., Cantú, C., González, H., \& Jiménez, J. (2014). Coniferas de Nuevo León, México. Linares, N. L., México: Universidad Autónoma de Nuevo León.

Fang, Z., Borders, E., \& Bailey, R. (2000). Compatible volume-taper models for Loblolly and Slash pine based on a system with segmented-stem form factors. Forest Science, 46(1), 1-12. doi: $10.1093 /$ forestscience/46.1.1

Fierros, R., \& Martinez, L. (2013). Modelos compatibles de ahusamiento y volumen para Pinus cooperiy Pinus engelmannii en la Región de San Dimas Durango. Tesis de Licenciatura. Instituto Tecnológico de El Salto. El Salto, Durango, México.

Flores-Morales, E. A., Aguirre-Calderón, O. A., Quiñónez-Barraza, G., González-Tagle, M. A., \& Jiménez-Pérez, J. (2019). Estimación del diámetro normal, altura y volumen de Pinus pseudostrobus Lindl. en función del diámetro del tocón. Revista Mexicana de Ciencias Forestales, 10(55), 154-170. doi. 10.29298/rmcf.v10i55.547

García, G., Aguirre, Ó., Vargas, B., Martínez, L., García, J., \& Hernández, J. (2019). Sistema compatible de ahusamiento y volumen comercial de Pinus pseudostrobus Lindl. en Nuevo San Juan Parangaricutiro, Michoacán, México. Agrociencia, 53(1), 115131.

Gómez-García, E., Diéguez-Aranda, U., Ózcelik, R., Sal-Cando, M., Castedo-Dorado, F., Crecente-Campo, F., Corral-Rivas, J., \& Arias-Rodil, M. (2016). Desarrollo de una función de perfil mediante modelos mixtos para Pinus sylvestris en Turquía: selección de parámetros fijos a expandir. Bosque (Valdivia), 37(1), 159-167. doi: 10.4067/S0717-92002016000100015

Harvey, W., R. (1975). Least squares analysis of data with unequal subclass numbers. Washington, DC: US Department of Agriculture. Agricultural Research Service.

Hernández-Ramos, J., Hernández-Ramos, A., García-Cuevas, X., Martínez-Ángel, L., Tamarit Urias, J., \& García Espinoza, G. (2018). Sistema compatible de ahusamiento-volumen comercial para Swietenia macrophylla King (caoba) en Quintana Roo, México. Madera y Bosques, 24(3). doi: 10.21829/myb.2018.2431441

Hernández-Ramos, J., de los Santos-Posadas, Héctor., Valdez-Lazalde, J., Tamarit-Urias, J., Ángeles-Pérez, G., Hernández-Ramos, A., Peduzzi, A., \& Carrero, O. (2017a). Sistema compatible de 
ahusamiento y volumen comercial para plantaciones de Eucalyptus urophylla en Tabasco, México. Acta Universitaria, 27(6), 40-52. doi: 10.15174/au.2017.1484

Hernández-Ramos, J., Hernández-Ramos, A., García-Magaña, J., GarcíaCuevas, X., García-Espinoza, G., Muñoz-Flores, H., \& OlveraDelgadillo, E. (2017b). Sistema compatible de ahusamientovolumen comercial para plantaciones de Pinus greggii Engelm. en Hidalgo, México. Revista Mexicana de Ciencias Forestales, 8(39), 5970. doi: $10.29298 /$ rmcf.v8i39.43

Jiang, L., Brooks, J., \& Wang., J. (2005). Compatible taper and volume equations for yellow-poplar in West Virginia. Forest Ecology and Management, 21(3), 399-409. doi: 10.1016/j.foreco.2005.04.006

Linnell, A. (1996). Analysis of repeated measures and time series. An introduction with forestry examples. Working paper. British Columbia Ministry of Forests.

López, G., Molina, P., Barrios, A., \& Aguirre, A. (2018). Modelos compatibles de ahusamiento-volumen para árboles de Gmelina arborea Roxb. en el Alto Magdalena, Colombia. Colombia Forestal, 21(2), 174-187. .doi: 10.14483/2256201X.12249

López, M. (2013). Modelos de ahusamiento y volumen comercial para Pinus oocarpa y Pinus douglasiana en la región de Pueblo Nuevo, Durango. Tesis de Maestría. Instituto Tecnológico de El Salto. El Salto, Durango, México.

Lynch, T., Chang, S., \& Chandler, J. (1992). Estimation of individual tree volume by importance sampling and antithetic variates from the cylindrical shells integral. Canadian Journal of Forest Research, 22(3), 326-335. doi: 10.1139/x92-042

Martínez-Ángel, L., De los Santos Posadas, H., González, A. F., CruzCobos, F., \& Quiñones-Barraza, G. (2019). Funciones compatibles de ahusamiento y volumen en una plantación forestal comercial de Pinus chiapensis (Martínez) Andresen en Tlatlauquitepec, Puebla. Agrociencia, 53(3), 381-401.

Max, T., \& Burkhart, H. (1976). Segmented polynomial regression applied to taper equations. Forest Science, 22(3), 283-289. doi: 10.1093 / forestscience/22.3.283

McTague, J., \& Bailey, R. (1987). Simultaneous total andmerchantable volume equations and a compatible taper function for loblolly pine. Canadian Journal of Forest Research, 17(1), 87-92. doi: $10.1139 / \mathrm{x} 87-015$

Parresol, B., Hotvedt, J., \& Cao, Q. (1987). A volume and taper prediction system for bald cypress. Canadian Journal of Forest Research, 17(3), 250-259. doi: 10.1139/x87-042

Pérez, L., \& Kannien, D. (2003). Provisional equations for estimating total and merchantable volume for Tectona grandis trees in Costa Rica. Forests, Trees and Livelihoods, 13(4), 345-359.
Pompa, M., Corral, J., Díaz, M., \& Martínez, M. (2009). Función de ahusamiento y volumen compatible para Pinus arizonica Engelm. En el suroeste de Chihuahua. Revista Mexicana de Ciencias Forestales, 34(105), 119-136.

Prodan, M., Peters, R., Cox, F., \& Real, P. (1997). Mensura Forestal. San José, Costa Rica: IICA-GTZ0.

Quiñonez-Barraza, G., De los Santos-Posadas, D., Héctor, M., ÁlvarezGonzález, J. G., \& Velázquez-Martínez, A. (2014). Sistema compatible de ahusamiento y volumen comercial para las principales especies de Pinus en Durango, México. Agrociencia, 48(5), 553-567.

Quiñonez-Barraza, G., García-Espinoza, G., \& Aguirre-Calderón, O. (2018). ¿Cómo corregir la heterocedasticidad y autocorrelación de residuales en modelos de ahusamiento y crecimiento en altura? Revista Mexicana de Ciencias Forestales, 9(49), 28-59. doi: 10.29298/rmcf.v9i49.151

Statistical Analysis System Institute Inc. (SAS). 2013. User's Guide. Version 9.4 for Windows. Cary, NC, USA.

Tamarit-Urias, J. C., Rojas-Díaz, E., Quiñonez-Barraza, G., OrdoñezPrado, C., \& Monárrez- González, J. (2017). Sistema de cubicación para árboles individuales de Quercus sp. en bosques bajo manejo de Puebla, México. Revista Mexicana de Ciencias Forestales, 8(40), 69-88. doi: 10.29298/rmcf.v8i40.37

Tamarit-Urias, J., De los Santos-Posadas, H., Aldrete, A., ValdezLazalde, J., Ramírez -Maldonado, H., \& Guerra-De la Cruz, V. (2014). Sistema de cubicación para árboles individuales de Tectona grandis L. f. mediante funciones compatibles de ahusamientovolumen. Revista Mexicana de Ciencias Forestales, 5(21), 58-74.

Torres, J., \& Magaña, S. (2001). Evaluación de plantaciones forestales. México: LIMUSA.

Unidad de Conservación y Desarrollo Forestal No. 1 [UCDF]. (1996). Programa de manejo forestal del ejido Pablillo municipio de Galeana, N. L. Galeana, Nuevo León.

Zakrzewski, W., \& MacFarlane, D. (2006). Regional stem profile model for cross-border comparisons of harvested red pine (Pinus resinosa Ait.) in Ontario and Michigan. Forest Science, 52(4), 468-475. doi: 10.1093 /forestscience/52.4.468

Manuscrito recibido el 20 de febrero de 2020

Aceptado el 19 de junio de 2020

Publicado el 5 de noviembre de 2021 
Este documento se debe citar como:

Flores M., E. A., Rodríguez A., A. C., Aguirre C., O. A., Alanís R., E., \& Quiñonez G., G. (2021). Sistema compatible de ahusamiento-volumen para Pinus pseudostrobus Lindl. en el ejido Corona del Rosal, Nuevo León, México. Madera y Bosques, 27(2), e2722130. doi: 10.21829/myb.2021.2722130

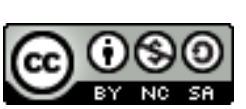

Madera y Bosques por Instituto de Ecología, A.C. se distribuye bajo una Licencia Creative Commons Atribución-NoComercialCompartirlgual 4.0 Internacional. 